\title{
Aplicação da técnica por cromatografia a gás para investigação da formação de sub produtos da desinfecção em água potável
}

\author{
Application of gas chromatographic technique for investigation \\ of the formation of by products in drinking water disinfection
}

Cristina Filomêna Pereira Rosa Paschoalato

Engenheira Química. Mestre e Doutora em Engenharia Hidráulica e Saneamento pela Escola de Engenharia de São Carlos da Universidade de São Paulo (EESC/USP) - São Carlos (SP), Brasil. Docente e pesquisadora da Universidade de Ribeirão Preto (UNAERP) - Ribeirão Preto (SP), Brasil.

\section{Genuino Rosário}

Aluno do Programa de Mestrado em Tecnologia Ambiental da UNAERP - Ribeirão Preto (SP), Brasil.

\section{Carmen Silvia Gonçalves Lopes}

Graduada em Química Industrial. Mestre em Química Orgânica pela Faculdade de Filosofia, Ciências e Letras de Ribeirão Preto da Universidade de São Paulo (FFCLRP/USP) - Ribeirão Preto (SP), Brasil. Doutoranda em Engenharia de Produção pela EESC/USP - São Carlos (SP), Brasil. Coordenadora e Docente do Curso de Engenharia Química da UNAERP - Ribeirão Preto (SP), Brasil.

\section{Márcia Maísa Freitas Afonso}

Graduada em Engenharia Química. Mestre em Química Orgânica e Doutora em Ciências pela FFCLRP/USP. Docente e Pesquisadora do Curso de Engenharia Química da UNAERP - Ribeirão Preto (SP), Brasil.

\section{Bruno Moreira da Silva}

Aluno de Iniciação Científica do Curso de Engenharia Química da UNAERP - Ribeirão Preto (SP), Brasil.

Talita Rafaella Silva Boldrin Dias

Aluna de Iniciação Científica do Curso de Engenharia Química da UNAERP - Ribeirão Preto (SP), Brasil.

\section{Resumo}

O uso do cloro para a desinfecção e/ou oxidação nas estações de tratamento de água favorece a formação de subprodutos orgânicos halogenados (SOH), muitos deles carcinogênicos. O objetivo desta pesquisa foi validar uma metodologia analítica proposta para a quantificação simultânea de 12 subprodutos da desinfecção por cromatografia a gás com detector de captura de elétrons (CG-DCE). O método apresentou linearidade ( $>00,998)$, repetibilidade menor que 0,15\%, limites de detecção de 1 a $6 \mu \mathrm{g} \cdot \mathrm{L}^{-1}$ e de quantificação de 3 a $21 \mu \mathrm{g} \cdot \mathrm{L}^{-1}$, precisão (<10\%), exatidão (93-120\%) e recuperação (87-117\%). Os resultados indicam que o método pode ser empregado para a investigação de $\mathrm{SOH}$ em água, garantindo confiabilidade analítica nos resultados.

Palavras-chave: compostos orgânicos halogenados; cromatografia a gás; outros subprodutos da desinfecção; subprodutos da desinfecção; trialometanos.

\begin{abstract}
The use of chlorine for disinfection and/or oxidation at drinking water treatment plants favors the formation of halogenated organic by-products (HOB), many of them carcinogenic. The purpose of this research was to validate an analytical method for the simultaneous quantification of $12 \mathrm{HOB}$ from disinfection by gas chromatograph with electrons capture detector (GC-ECD). The method provided linearity ( $r>0.998$ ), repeatability lower than $0.15 \%$, limits of detection from 1 to $6 \mu \mathrm{g} \cdot \mathrm{L}^{-1}$ and of quantification from 3 to $21 \mu \mathrm{g} \cdot \mathrm{L}^{-1}$, precision ( $\left.<10 \%\right)$, accuracy $(93-120 \%)$ and recovery $(87-117 \%)$. The results indicate that the method can be employed to investigate the formation of $\mathrm{HOB}$ in water, yielding analytically reliable results.
\end{abstract}

Keywords: halogenated organic compounds; gas chromatograph; other disinfection by-products; disinfection by-products; trihalometanes 


\section{Introdução}

Os principais subprodutos da desinfecção que podem ser formados nos sistemas de tratamento de água potável são: trialometanos (THM), ácidos haloacéticos (AHA), haloacetonitrilas, haletos cianogênicos, halopicrinas, haloacetonas, haloaldeídos, halofenóis, mx [3-cloro-4-(diclorometil)-5-hidroxi-2(5h)-furanona] (ROOK \& EVANS, 1974), entre outros, não contemplados no padrão de potabilidade da Portaria MS no 2.914 (BRASIL, 2011), que têm sido detectados em águas cloradas (PASCHOALATO; TRIMAILOVAS; DI BERNARDO, 2008; DANTAS et al., 2008).

A United States Environmental Protection Agency (USEPA) aprovou e publicou os primeiros métodos analíticos para quantificação dos subprodutos orgânicos halogenados $(\mathrm{SOH})$ por meio da cromatografia a gás com detector de captura de elétrons (CG-DCE) (KRASNER et al., 1989; FAIR \& BOLYARD, 1991). Posteriormente, ABNT (1995), APHA, AWWA e WEF (2005) e Xie (2003) publicaram métodos analíticos para determinação de trialometanos e outros subprodutos.

A técnica analítica de CG-DCE, principalmente para quantificação de THM, vem sendo utilizada com sucesso para controle, redução e remoção de subprodutos da desinfecção (FERREIRA FILHO; HESPANHOL; PIVELI, 2003; PASCHOALATO, 2005; DANTAS et al., 2008; ALVARENGA, 2010; CARVALHO, 2010; AGRIZZI, 2011).

Considerando-se a importância da investigação e do controle de compostos carcinogênicos, faz-se necessário um estudo analítico, por meio da validação de uma metodologia por CG-DCE, para quantificação simultânea de outros subprodutos da desinfecção, possíveis de serem encontrados em águas de abastecimento.

\section{Metodologia}

O procedimento analítico foi baseado nas recomendações do método 551.1 (USEPA, 1995). O equipamento utilizado foi um cromatógrafo a gás modelo 3600 CX (Varian) com detector de captura de elétrons (CG-DCE). A aquisição de dados foi realizada por um microcomputador com o software para cromatografia Galaxie WS Ver. 1.9. O gás de arraste foi o nitrogênio 5.0 DCE (White Martins) e o sistema injetor foi do tipo slit/splitless acoplado a uma coluna DB-1, com $30 \mathrm{~m}$ de comprimento, 0,32 $\mathrm{mm}$ de diâmetro interno e $5 \mu \mathrm{m}$ de filme (Agilent J\&W Scientific).

Para a calibração, foram adquiridas as seguintes soluções: padrão misto de trialometanos (48140-U, Supelco); padrão misto de halogenados voláteis para haloacetonitrilas, cloropicrina e haloacetonas (EPA 551B 4-8046, Supelco) e padrão puro de cloro hidrato (47335-U, Supelco). O composto utilizado como padrão interno (PI) foi o diclorometano grau cromatográfico (J.T. Baker) e o solvente utilizado na extração foi o metil terc-butílico éter (MTBE) com grau de pureza cromatográfica. Para a diluição dos padrões, foi preparada uma água ultrapura, obtida por purificador da marca Milli-Q e submetida a fervura por 10 minutos, exposição à radiação UV por 1 minuto, borbulhamento de nitrogênio por 5 minutos e resfriamento, sendo finalmente acondicionada em frasco de vidro escuro e conservada a $4^{\circ} \mathrm{C}$, para garantir a isenção de compostos voláteis, cloro residual e matéria orgânica.

As soluções-padrão foram diluídas em acetona de pureza grau cromatográfico (J.T. Baker), exceto o padrão de cloro hidrato, que foi diluído em metanol para evitar degradação (USEPA, 1995). A partir dessas soluções, foram preparadas diferentes soluções de padrões diluídas em água ultrapura, obtendo-se concentrações crescentes em balões volumétricos de $10 \mathrm{~mL}$.

Para as análises de compostos orgânicos por cromatografia a gás, são necessários procedimentos de extração. A USEPA (1995) recomenda a técnica de extração do tipo líquido-líquido com uso de MTBE; já outras fontes recomendam o uso de n-pentano (ABNT, 1995) ou hexano (APHA; AWWA; WEF, 2005). Alternativamente outras técnicas de extração podem ser empregadas, tais como: injeção aquosa direta, microextração em fase líquida, técnicas de headspace (estático, microextração em fase sólida e purge and trap) e técnicas de extração usando membranas (PAVÓN et al., 2008; GOLFINOPOULOS; LEKKAS; NIKOLAOU, 2001; TOR \& AYDIN, 2006; CHO; KONG; OH, 2003).

Nesta metodologia, foi utilizada a extração líquido-líquido. $\mathrm{O}$ procedimento iniciou-se com a adição de $10 \mu \mathrm{L}$ de padrão interno diclorometano 1\% p/v em metanol, em balões volumétricos contendo $10 \mathrm{~mL}$ de solução padrão de diferentes concentrações, inclusive em um branco. Em seguida, transferiu-se o conteúdo total dos balões para um frasco de vidro com tampa. A extração foi processada com adição de $10 \mathrm{~mL}$ de MTBE a $4^{\circ} \mathrm{C}$ em cada frasco, submetidos a agitação (100 rpm) por 30 minutos, seguida de resfriamento a $4^{\circ} \mathrm{C}$, obtendo-se a separação de duas fases; $5 \mathrm{~mL}$ da fase orgânica foi retirado e transferido para um frasco de vidro, onde se adicionou aproximadamente $1 \mathrm{mg}$ de sulfato de sódio anidro para adsorção de umidade. Em seguida, os frascos contendo os extratos foram acondicionados em freezer $\left(-4^{\circ} \mathrm{C}\right)$, para posterior injeção de $1 \mu \mathrm{L}$ no CG-DCE.

$\mathrm{Na}$ aplicação da metodologia para investigação dos subprodutos da desinfecção em amostras de água (matriz real), deve-se adotar o mesmo procedimento analítico de extração, considerando-se o volume de amostra igual ao da solução padrão (10 mL). No caso de água clorada, é necessária a prévia descloração com adição de, aproximadamente, $6 \mathrm{mg}$ de acido ascórbico por $100 \mathrm{~mL}$ de amostra (USEPA, 1995) ou, alternativamente, tiossulfato de sódio ou sulfito de sódio (APHA; AWWA; WEF, 2005). No procedimento utilizado para a validação, foram avaliadas a seletividade, a linearidade, a repetibilidade, os limite de detecção e o limite de quantificação, recuperação, precisão e exatidão (LANÇAS, 2004; INMETRO, 2007).

A seletividade do método foi obtida por meio da otimização das seguintes condições analíticas: fase móvel; temperatura da coluna; fase estacionária; rampa de aquecimento; temperatura do injetor e 
detector e fluxo de gases; já a linearidade foi obtida por curvas analíticas de calibração com concentrações crescentes, utilizando-se as soluções provenientes do procedimento de extração. Esses resultados possibilitam um tratamento estatístico por regressão linear para a obtenção do coeficiente de correlação $(r)$, sendo satisfatório que o mesmo seja maior que 0,99 (ANVISA, 2003).

A repetibilidade (r\%) representa a fidelidade quando as condições operacionais forem realizadas pelo mesmo analista, no mesmo equipamento e em um curto intervalo de tempo (LANÇAS, 2004). O parâmetro utilizado foi o tempo de retenção de cada analito. Foram efetuadas seis injeções sucessivas de uma mesma solução de água fortificada com adição de padrão, onde a mesma passou pelo procedimento. A partir de um tratamento estatístico, calculou-se o valor de r(\%), utilizando-se a Equação 1 e considerando-se como aceitáveis os valores menores que 1\% (ANVISA, 2003).

$\mathrm{r} \%=\mathrm{t} \times S_{\mathrm{r}} \mathrm{x} \sqrt{2}$

onde:

r\%: repetibilidade;

t: valor obtido da tabela de Student;

$\mathrm{S}_{\mathrm{r}}$ : desvio padrão da repetibilidade.

O limite de detecção (LD) corresponde à menor quantidade detectada de um analito, porém não necessariamente quantificada como um valor exato. O limite de quantificação (LQ) é a menor quantidade de analito que pode ser quantificada com exatidão e com uma fidelidade determinada. O LD e o LQ foram obtidos a partir dos resultados de linearidade, considerando-se a estatística de regressão linear de cinco curvas analíticas de calibração, obtidas para cada composto, conforme as Equações 2 e 3, respectivamente.

$\mathrm{LD}=\frac{\mathrm{DP}_{\mathrm{a}} \times 3}{\mathrm{IC}}$

$\mathrm{LQ}=\frac{\mathrm{DP}_{\mathrm{a}} \times 10}{\mathrm{IC}}$

onde:

$\mathrm{DP}_{\mathrm{a}}$ : desvio padrão da intercepção com o eixo y;

IC: inclinação da reta obtida da curva analítica de calibração (LANÇAS, 2004)

A recuperação visa avaliar a eficiência do processo de extração do analito considerando-se a matriz na qual se encontra, neste caso, a água. Uma solução de concentração conhecida da mistura de todos os analitos foi preparada em meio aquoso $(\mathrm{Cl})$ e em meio orgânico com MTBE (C2). A mistura em meio aquoso foi extraída e, em seguida, ambas foram injetadas no CG-DCE. A eficiência da recuperação na extração foi calculada pela Equação 4, sendo que os valores aceitos encontram-se na faixa de 70 a 120\% (LANÇAS, 2004).
Recuperação $(\%)=\frac{C 1}{C 2} \times 100$

A precisão demonstra a dispersão entre os resultados analíticos obtidos repetidamente de uma mesma solução padrão e a exatidão expressa pela concordância entre o valor encontrado e o valor aceito como verdadeiro ou como referência e está sempre associado a valores de precisão (LANÇAS, 2004). A precisão foi determinada pelo cálculo do desvio padrão relativo (DPR) e da exatidão, por meio da relação entre a concentração média experimental (CME) e a concentração teórica (CT), previamente conhecida, conforme as Equações 5 e 6 , respectivamente. Foram preparadas três soluções de padrão em diferentes concentrações - contendo a mistura de todos os analitos dissolvidas em água. Cada uma delas foi extraída e injetada seis vezes $(n=6)$ no CG-DCE

$\mathrm{DPR}=\frac{\mathrm{DP}}{\mathrm{CME}} \times 100$

onde:

DP: desvio padrão

Exatidão $=\frac{C M E}{C T} \times 100$

\section{Resultados e discussão}

As condições operacionais cromatográficas que resultaram na separação e na identificação de 12 analitos e do padrão interno foram: fluxo do gás de arraste de 3,6 mL.min ${ }^{-1}$, pressão de 15 psi; injetor na temperatura de $160^{\circ} \mathrm{C}$ e detector a $290^{\circ} \mathrm{C}$; razão de divisão de fluxo (split) de 2:1; temperatura do forno da coluna a $40^{\circ} \mathrm{C}$ por 1 minuto, com rampas de aquecimento de $5^{\circ} \mathrm{C} \cdot \mathrm{min}^{-1}$ até $125^{\circ} \mathrm{C}$ e de $20^{\circ} \mathrm{C} \mathrm{min}{ }^{-1}$ até $180^{\circ} \mathrm{C}$, permanecendo por 5 minutos. A Figura 1 mostra o cromatograma de uma água ultrapura com uma mistura dos padrões, obtendo-se estabilidade da linha de base, identificação do padrão interno e dos 12 analitos de interesse, todos com tempo de retenção não coincidentes e áreas bem definidas.

Na Tabela 1, apresentam-se os valores das faixas de concentrações dos analitos, os valores de coeficiente linear ( $r$ ), dos limites de detecção e de quantificação em $\mu \mathrm{g} . \mathrm{L}^{-1}$, além dos resultados de recuperação, sendo um inferior (P2), um intermediário (P4) e um superior (P6).

As diferentes faixas de concentração de padrões mistos, conforme a Tabela 1, apresentaram valores de coeficiente linear de 0,9984 a 0,9997, demonstrando linearidade adequada. Os limites de detecção e de quantificação foram obtidos por meio dos cálculos das regressões lineares aplicados às Equações 2 e 3 .

Os valores obtidos entre 87 e $117 \%$ para a recuperação satisfizeram a recomendação de 70 a 120\%, demostrando que a técnica empregada de extração foi eficiente e confiável. O mesmo aconteceu 


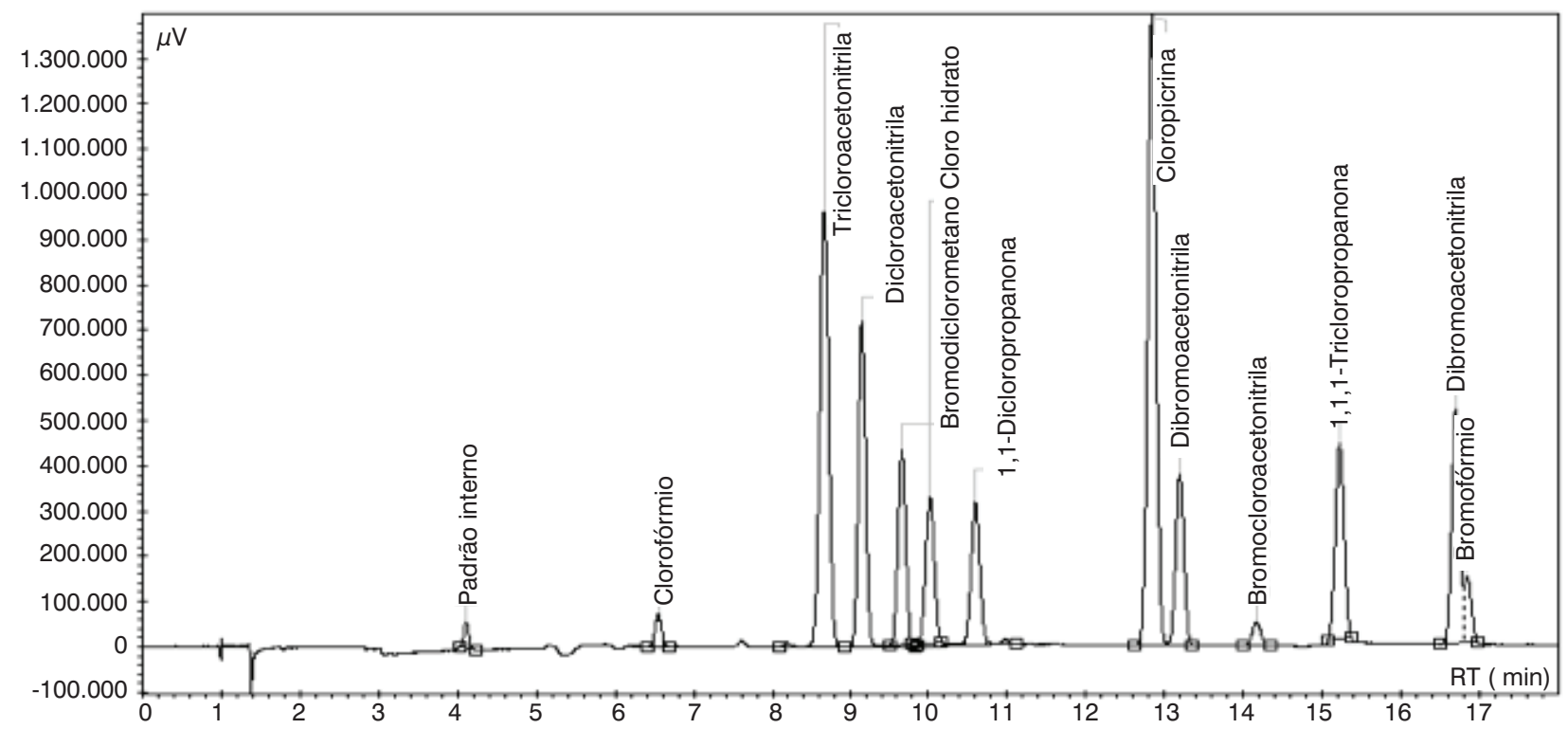

Figura 1 - Cromatograma de uma água ultrapura fortificada com mistura de padrões e padrão interno nas condições otimizadas.

Tabela 1 - Faixas de concentração das curvas analíticas, resultados de coeficiente linear, limites de detecção, limite de quantificação e recuperação para os pontos P2, P4 e P6.

\begin{tabular}{|c|c|c|c|c|c|c|c|}
\hline \multirow{2}{*}{ Analito } & \multirow{2}{*}{$\begin{array}{c}\text { Faixa } \\
\left(\mu \mathrm{g} \cdot \mathrm{L}^{-1}\right)\end{array}$} & \multirow{2}{*}{$\begin{array}{c}\text { Coeficiente } \\
\text { linear }(r)\end{array}$} & \multicolumn{2}{|c|}{ Limites } & \multicolumn{3}{|c|}{ Recuperação } \\
\hline & & & Detecção $\left(\mu \mathrm{g} \cdot \mathrm{L}^{-1}\right)$ & Quantificação ( $\left.\mu \mathrm{g} \cdot \mathrm{L}^{-1}\right)$ & P2 (\%) & P4 (\%) & P6 (\%) \\
\hline Clorofórmio & 2,5 a 100 & 0,9995 & 4 & 13 & 108 & 101 & 98 \\
\hline Tricloroacetonitrila & 2,5 a 120 & 0,9992 & 2 & 7 & 98 & 91 & 97 \\
\hline Dicloroacetonitrila & 2,5 a 120 & 0,9993 & 2 & 6 & 112 & 88 & 90 \\
\hline Bromodiclorometano & 2,5 a 100 & 0,9984 & 3 & 9 & 101 & 93 & 87 \\
\hline Cloro hidrato & 2,5 a 100 & 0,9994 & 5 & 17 & 101 & 100 & 106 \\
\hline 1,1-Dicloropropanona & 2,5 a 120 & 0,9993 & 1 & 4 & 104 & 105 & 107 \\
\hline Cloropicrina & 2,5 a 120 & 0,9997 & 2 & 7 & 106 & 89 & 98 \\
\hline Dibromoclorometano & 2,5 a 100 & 0,9990 & 3 & 11 & 104 & 93 & 89 \\
\hline Bromocloroacetonitrila & 2,5 a 120 & 0,9992 & 1 & 3 & 113 & 117 & 96 \\
\hline 1,1,1-Tricloropropanona & 2,5 a 120 & 0,9993 & 5 & 17 & 110 & 93 & 109 \\
\hline Dibromoacetonitrila & 2,5 a 120 & 0,9992 & 2 & 7 & 113 & 93 & 103 \\
\hline Bromofórmio & 2,5 a 100 & 0,9993 & 6 & 21 & 104 & 94 & 95 \\
\hline
\end{tabular}

com os valores de repetibilidade entre 0,02 e 0,14\%, que estão de acordo com o valor aceitável, que é menor que 1\%.

A Tabela 2 contém os resultados da repetibilidade em função do tempo de retenção, com média de seis injeções, desvio padrão e valor de percentual de repetibilidade obtidos para três pontos da curva: início (P1), intermediário (P4) e final (P7).

A precisão e a exatidão foram avaliadas em três diferentes pontos da curva de calibração e os resultados estão representados na Tabela 3.

Os valores de precisão, expressos pelo DPR, ficaram entre 2 e 13\%. Já para a exatidão, os resultados ficaram entre 93 e 117\%. O método mostrou-se preciso e exato, visto que a literatura recomenda desvio máximo de 20\% (ANVISA, 2003).

\section{Conclusão}

A metodologia proposta apresentou resultados que atendem às normas de validação analítica, podendo ser empregada com confiabilidade na investigação da formação de trialometanos e também de outros subprodutos da oxidação com cloro e seus derivados em água, que, mesmo não estando contemplados no atual padrão de potabilidade brasileiro, podem apresentar risco à saúde e potencializar a formação de trialometanos e ácidos haloacéticos. Nesse sentido, a referida metodologia deverá auxiliar pesquisadores e técnicos do setor de tratamento de água, visto que possibilita a quantificação simultânea de 12 subprodutos, de maneira simples e rápida, por meio de um único equipamento de CG-DCE. 
Tabela 2 - Resultados de repetibilidade em função do tempo de retenção das amostras de água ultrapura fortificada com diferentes concentrações (P1, P4 e P7), com média ( $n=6)$ e desvio padrão.

\begin{tabular}{|c|c|c|c|c|c|c|c|c|c|}
\hline \multirow[b]{2}{*}{ Analito } & \multicolumn{3}{|c|}{ P1 } & \multicolumn{3}{|c|}{ P4 } & \multicolumn{3}{|c|}{ P7 } \\
\hline & $\begin{array}{c}\text { Média } \\
\text { (min) }\end{array}$ & $S_{r}$ & $(\mathrm{r} \%)$ & $\begin{array}{l}\text { Média } \\
\text { (min) }\end{array}$ & $S_{r}$ & $(\mathrm{r} \%)$ & $\begin{array}{c}\text { Média } \\
\text { (min) }\end{array}$ & $S_{r}$ & $(\mathrm{r} \%)$ \\
\hline Diclorometano (PI) & 4,11 & 0,0133 & 0,0376 & 4,11 & 0,0151 & 0,043 & 4,10 & 0,0084 & 0,024 \\
\hline Clorofórmio & 6,55 & 0,0293 & 0,0828 & 6,55 & 0,0315 & 0,089 & 6,53 & 0,0137 & 0,039 \\
\hline Tricloroacetonitrila & 8,68 & 0,0279 & 0,0788 & 8,68 & 0,0349 & 0,099 & 8,66 & 0,0152 & 0,043 \\
\hline Dicloroacetonitrila & 9,16 & 0,0293 & 0,0828 & 9,16 & 0,0349 & 0,099 & 9,13 & 0,0155 & 0,044 \\
\hline Bromodiclorometano & 9,67 & 0,0301 & 0,0852 & 9,66 & 0,0372 & 0,105 & 9,64 & 0,0137 & 0,039 \\
\hline Cloro hidrato & 10,02 & 0,0310 & 0,0876 & 10,02 & 0,0422 & 0,119 & 9,99 & 0,0137 & 0,039 \\
\hline 1,1-Dicloropropanona & 10,61 & 0,0321 & 0,0908 & 10,60 & 0,0392 & 0,111 & 10,57 & 0,0155 & 0,044 \\
\hline Cloropicrina & 12,85 & 0,0394 & 0,1114 & 12,84 & 0,0450 & 0,127 & 12,81 & 0,0167 & 0,047 \\
\hline Dibromoclorometano & 13,20 & 0,0360 & 0,1018 & 13,20 & 0,0432 & 0,122 & 13,16 & 0,0163 & 0,046 \\
\hline Bromocloroacetonitrila & 14,17 & 0,0392 & 0,1109 & 14,17 & 0,0512 & 0,145 & 14,13 & 0,0147 & 0,042 \\
\hline 1,1,1-Tricloropropanona & 15,23 & 0,0418 & 0,1182 & 15,23 & 0,0524 & 0,148 & 15,19 & 0,0110 & 0,031 \\
\hline Dibromoacetonitrila & 16,70 & 0,0450 & 0,1273 & 16,70 & 0,0504 & 0,142 & 16,66 & 0,0151 & 0,043 \\
\hline Bromofórmio & 16,86 & 0,0467 & 0,1320 & 16,85 & 0,0496 & 0,140 & 16,81 & 0,0155 & 0,044 \\
\hline
\end{tabular}

r\%: repetibilidade; $\mathrm{S}_{\mathrm{r}}$ : desvio padrão de repetibilidade.

Tabela 3 - Resultados de precisão e exatidão obtidos para os pontos P1, P4 e P7 da curva de calibração.

\begin{tabular}{|c|c|c|c|c|c|c|}
\hline \multirow{2}{*}{ Analito } & \multicolumn{2}{|c|}{ P1 } & \multicolumn{2}{|c|}{ P4 } & \multicolumn{2}{|c|}{ P7 } \\
\hline & Precisão (DPR) & Exatidão (\%) & Precisão (DPR) & Exatidão (\%) & Precisão (DPR) & Exatidão (\%) \\
\hline Clorofórmio & 9 & 96 & 2 & 99 & 13 & 93 \\
\hline Tricloroacetonitrila & 7 & 117 & 3 & 102 & 1 & 100 \\
\hline Dicloroacetonitrila & 6 & 115 & 3 & 104 & 3 & 101 \\
\hline Bromodiclorometano & 6 & 104 & 3 & 105 & 3 & 104 \\
\hline Cloro Hidrato & 6 & 101 & 9 & 114 & 10 & 111 \\
\hline 1,1-Dicloropropanona & 7 & 112 & 3 & 105 & 3 & 102 \\
\hline Cloropicrina & 7 & 116 & 2 & 102 & 2 & 101 \\
\hline Dibromoclorometano & 6 & 105 & 2 & 104 & 3 & 105 \\
\hline Bromocloroacetonitrila & 11 & 110 & 3 & 104 & 6 & 101 \\
\hline 1,1,1-Tricloropropanona & 7 & 117 & 10 & 107 & 4 & 110 \\
\hline Dibromoacetonitrila & 7 & 116 & 3 & 103 & 1 & 102 \\
\hline Bromofórmio & 7 & 106 & 5 & 103 & 2 & 102 \\
\hline
\end{tabular}

DPR: desvio padrão relativo.

\section{Referências}

ABNT - Associação Brasileira de Normas Técnicas (1995) Norma Brasileira Regulamentadora NBR-13407. Água - Determinação de trialometanos em água tratada para abastecimento por extração líquido/ líquido. Rio de Janeiro: ABNT.

AGRIZZI, A.D. (2011) Avaliação da formação de trialometanos considerando o uso de cloro e permanganato de potássio como pré-oxidante em água de abastecimento Dissertação (Mestrado em Engenharia Ambiental) - Universidade Federal do Espírito Santo, Fundo de apoio da Ciência e Tecnologia de Vitória, Vitória.

ALVARENGA, J.A. (2010) Avaliação da formação de subprodutos da cloração em água para consumo humano. Dissertação (Mestrado em Saneamento, Meio Ambiente e Recursos Hídricos) - Universidade Federal de Minas Gerais, Belo Horizonte.

ANVISA - Agência Nacional de Vigilância Sanitária (2003) Resolução $(R E) n^{\circ} 899$, de 29 de maio de 2003. Guia para validação de métodos analíticos e bioanalíticos. Brasil: Ministério da Saúde.
APHA - American Public Health Association; AWWA - American Water Works Association; WEF - Water Environment Federation (2005) Standard methods for the examination of water and wastewater. $21^{\text {st }} \mathrm{ed}$. Washington DC: APHA, AWWA, WEF.

BRASIL. Ministério da Saúde (2011) Portaria MS n².914, de 12 de dezembro de 2011. Dispõe sobre os procedimentos de controle e de vigilância da qualidade da água para consumo humano e seu padrão de potabilidade. Ministério da Saúde: Brasília.

CARVALHO, R.P.M. (2010) Remoção de saxitoxina por meio de oxidação com cloro livre. Tese (Doutorado em Tecnologia Ambiental e Recursos Hídricos) - Universidade de Brasília, Brasília.

CHO, D.H.; KONG, S.H.; OH, S.G. (2003) Analysis of trihalomethanes in drinking water using headspace-SPME technique with gas chromatography Water Research, v. 37, p. 402.

DANTAS, A.B.; PASCHOALATO, C.F.P.R.; MONTANHA, W.A.A.; TRIMAILOVAS, M.R. (2008) Influência da pré-oxidação com 
cloro e dióxido de cloro de água contendo substâncias húmicas aquáticas na coagulação-filtração e na formação de subprodutos orgânicos halogenados. Revista DAE, v. 22, p. 178.

FAIR, P.S. \& BOLYARD, M. (1991) Status of disinfectant/ disinfection by-product methods in relation to the disinfection by-products rules. In: Water Quality Technology Conference, Orlando: American Water Works Association (AWWA). p. 399-407.

FERREIRAFILHO, S.S.; HESPANHOL, I.; PIVELI, R.P. (2003) Aplicabilidade do Dióxido de Cloro no Tratamento de Águas de Abastecimento. In: Congresso Brasileiro de Engenharia Sanitária e Ambiental, 22 Anais... Joinville: ABES.

GOLFINOPOULOS, S.K.; LEKKAS, T.D.; NIKOLAOU, A.D. (2001) Comparison of methods for determination of volatile organic compounds in drinking water. Chemosphere, v. 45, p. 275

INMETRO - Instituto Nacional de Metrologia, Normalização e Qualidade Industrial (2007). Orientação sobre validação de métodos de ensaios químicos. DOQ-CGCRE-008. Revisão 2. São Paulo.

KRASNER, S.W.; MCGUIRE, M.J.; JACANGELO, J.G.; PATANIA, N.L.; REAGAN, K.M.; AIETA, M.E. (1989) The occurrence of disinfection by-products in U.S. drinking water. Journal American Water Works Association, v. 81, p. 41-53.

LANÇAS, F. (2004) Validação de métodos cromatográficos de análises. São Carlos: RiMa.
PASCHOALATO, C.F.P.R. (2005) Efeito da pré-oxidação, coagulação, filtração e pós-cloração na formação de subprodutos orgânicos halogenados em águas contendo substâncias húmicas. Tese (Doutorado) - Engenharia civil. Área de concentração Hidráulica e Saneamento. Escola de Engenharia de São Carlos, Universidade de São Paulo, São Carlos.

PASCHOALATO, C.F.P.R.; TRIMAILOVAS, M.R.; DI BERNARDO, L. (2008) Formação de subprodutos orgânicos halogenados nas operações de préoxidação com cloro, ozônio e peroxônio e pós-cloração em água contendo substância húmica. Engenharia Sanitária e Ambiental, v. 13, p. 313.

PAVÓN, J.L.P.; MARTÍN, S.H.; PINTO, C.G.; CORDERO, B.M. (2008) Determination of trihalomethanes in water samples. Analytica Chimica Acta, v. 6, p. 629.

ROOK, J. \& EVANS, S. (1974) Renoval of trihalometanos precursors from surface waters using weak resins. Journal American Water Works Association, v. 9, p. 520-524.

TOR, A. \& AYDIN, M.E. (2006) Application of liquid-phase microextraction to the analysis of trihalomethanes in water. Analytica Chimica Acta, v. 138, p. 575.

USEPA - United States Environmental Protection Agency (1995) Method 551.1. Determination of chlorination disinfection byproducts, chlorinated solvents, and halogenated pesticides/herbicides in drinking water by liquid-liquid extraction and gas chromatography with electron-capture detection. Revisão 1. Ohio: USEPA. CD-ROM.

XIE, Y.F. (2003) Disinfection byproducts in drinking water: formation, analysis, and control. Florida: CRC Press. 